\section{Dorsolateral prefrontal cortex and Stroop performance: Tackling the lateralization}

\author{
Marie-AnNe VANDERHASSElt AND Rudi De RAEDT \\ Ghent University, Ghent, Belgium
}

\author{
AND \\ Chris BaEken \\ University Hospital Brussels (UZ Brussel), Brussels, Belgium
}

Neuroscience research has identified the involvement of the dorsolateral prefrontal cortex (DLPFC) in cognitive control. Questions remain, however, about its lateralization correlates during Stroop task performance, an experimental cornerstone on which a large amount of cognitive control research is based. After reviewing the literature, we find that three Stroop variants have been used in an attempt to uncover different aspects of cognitive control related to DLPFC involvement. In sum, rapid and sequential up-regulation of the attentional set seems to be related to the left DLPFC. These attentional adjustments are based on participants' expectancies regarding the conflicting nature of the upcoming trial, and not on the conflict itself. In contrast, the right DLPFC is associated with an overall up-regulation of the attentional set when attentional conflict is experienced.

\section{Theoretical Background}

Cognitive control can be conceptualized as the capacity to suppress prepotent but incorrect responses and the ability to filter out irrelevant information within a stimulus set (Botvinick, Braver, Barch, Carter, \& Cohen, 2001). Experimental conflict situations that require cognitive control have been found in tasks that demand overriding of prepotent responses, such as in the Stroop (1935) paradigm. The Stroop interference effect is one of the most frequently used tasks in cognitive psychology, clinical neuropsychology, and cognitive neuroscience. In a Stroop color-naming task, participants have to name the ink color of a printed color word, and greater conflict occurs for incongruent (e.g., the word RED in green ink) than for congruent (e.g., the word RED in red ink) trials - in other words, the Stroop interference effect.

Neuroimaging techniques can be used to investigate the brain correlates of cognitive control during experiments. Such activation techniques include PET (positron emission tomography), fMRI (functional magnetic resonance imaging), and ERPs (event-related potentials). These neuroimaging techniques face the critical problem, though, of distinguishing cause from effect, because no interference with brain activity is provoked. As a noninvasive tool for stimulation of the human cerebral cortex, repetitive transcranial magnetic stimulation (rTMS) induces alterations of neuronal activity that have an effect on cognition; this is becoming a promising technique for investigating whether a particular area is essential for task performance.

Different neurocognitive studies have revealed that cognitive control is related to a specific cortico-subcortical circuit, including the anterior cingulate cortex (ACC) and the dorsolateral prefrontal cortex (DLPFC) (e.g., Barber \& Carter, 2005; MacDonald, Cohen, Stenger, \& Carter, 2000). According to the prominent conflict-monitoring hypothesis (Botvinick et al., 2001; Botvinick, Nystrom, Fissell, Carter, \& Cohen, 1999), the occurrence of response conflict is signaled by the ACC, leading to recruitment in the DLPFC of more cognitive control for subsequent performance. This involvement of the DLPFC (Brodmann area [BA] 9/46) has been identified in neuroscience research with top-down regulatory processes of cognitive control. Overall, BA 9 includes the superior and inferior frontal gyrus, whereas BA 46 includes the middle frontal gyrus.

For a number of years, the correlates of top-down attentional control were thought to reflect the integrity of the left frontal cortex and the cortico-subcortical circuit (Swick \& Jovanovic, 2002). However, recent literature has demonstrated a more heterogeneous picture of lateralization with respect to cognitive control and the DLPFC. Notably, the lateralization of control processes seems to be task-specific (Kerns et al., 2004; Miller \& Cohen, 2001; Rubia et al., 2006). Since the Stroop interference effect remains a cornerstone on which the conflict-monitoring model is based, this review article focuses on lateralized processes of cognitive control based on different Stroop designs that have been used.

Using a standard Stroop task with only a color-naming instruction and equal numbers of congruent and incongruent trials, one session of rTMS $(10 \mathrm{~Hz})$ over the left DLPFC (BA 9/46) had beneficial effects on both congruent and incongruent trials, whereas no changes occurred in the placebo sham condition (Vanderhasselt, De Raedt, Baeken, Leyman, \& D'haenen, 2006). Although this can be considered to be consistent with the hypothesis that the left DLPFC plays a role in the implementation of control, by representing and actively maintaining the attentional demands of the task (i.e., imposing an attentional set: Harrison et al., 2005), one could also argue that enhanced attentional control should lead to a decreased interference effect (MacDonald et al., 2000). Although rTMS had a larger effect on incongruent trials, the significant effect indicative of a decreased interference effect disappeared completely after the inclusion of order of stimulation (counterbalanced sham vs. rTMS) in the analysis.

On the other hand, using a similar Stroop task, fMRI research by Kerns et al. (2004) did not find left DLPFC (BA 9/46) activation related to cognitive control implementation. Although the same Stroop task was used in

M.-A.Vanderhasselt, marieanne.vanderhasselt@ugent.be 
both studies and both found DLPFC involvement, their research findings regarding lateralization contradict each other. However, different aspects of cognitive control could relate to processing in the left or the right DLPFC (Stürmer, Redlich, Irlbacher, \& Brandt, 2007).

After more then a decade of research, three Stroop variants have been shown to influence different aspects of cognitive control related to DLPFC involvement. First, a task-switching version of the Stroop task can be used, in which participants have to switch between color naming and word reading. Hence, after a color-naming instruction, the implementation of cognitive control increases because of preparatory conflict adaptation processes. Second, varying the ratio of congruent to incongruent trials during high-conflict (relatively more congruent trials) and lowconflict (relatively more incongruent trials) conditions also manipulates cognitive control. In low-conflict blocks, long-term strategic modification occurs in response to the high frequency of incongruent trials (macro-adjustments due to the context), which decreases conflict during incongruent trials. Finally, given the same number of congruent and incongruent trials, the interference effects following an incongruent trial are reduced, resulting in the so-called conflict adaptation effect (Gratton, Coles, \& Donchin, 1992). Because of increased cognitive control, responses are faster and more accurate after incongruent than after congruent trials.

The hemispheric specialization of the left and right DLPFC during Stroop performance has been briefly addressed in many articles, but the findings have never been integrated. Therefore, a review tackling the lateralization of the DLPFC in cognitive control using the Stroop task is presented here.

\section{Task-Switching Version of the Stroop Task}

A well-known fMRI study by MacDonald et al. (2000) demonstrated that in a task-switching version of the Stroop task, the left DLPFC (BA 46) was selectively active during the preparatory cue period for color naming, as compared with word reading; in the latter task, responses were more automatic. In addition, the increased left DLPFC activity was correlated with reduced interference effects. Most importantly, left DLPFC was not more activated during incongruent than during congruent color-naming responses. This implies that the left DLPFC is active when there is foreknowledge of upcoming conflict, leading to attentional preparation.

These findings are in accord with those from the lesion studies of Stuss, Floden, Alexander, Levine, and Katz (2001), which demonstrated that left DLPFC lesions were correlated with overall increases in reaction times (RTs) and errors in color naming, but not with a disproportionate increase in RTs or error rates during incongruent trials. Instead, patients with bilateral superior medial frontal cortical damage showed greater interference effects. This suggests that the left DLPFC involvement is not specifically related to conflict itself (e.g., on incongruent trials), but to a temporarily increased attentional set for reducing upcoming conflict.
Moreover, in a recent fMRI experiment, Aarts, Roelofs, and van Turennout (2008) presented informative and uninformative cues well before the Stroop target. An informative cue indicated whether the upcoming target was congruent, incongruent, or neutral. After informative cues, the left DLPFC (BA 46) was more active for incongruent than for congruent targets, demonstrating a regulatory strategy. This once again indicates that, when a participant has foreknowledge of an upcoming conflict, the left DLPFC becomes activated to increase a preparatory attentional set. These online representations are based on the fast recruitment of working memory to strategically prepare for conflict.

\section{Varying the Ratio of Congruent and Incongruent Trials}

Second, the amount of cognitive control can be manipulated by varying the ratio of congruent to incongruent trials during different blocks. Within this context, Milham, Banich, and Barad (2003) explored the role of the left DLPFC using a Stroop color-naming task with three types of infrequent (oddball) trials: a block with neutral oddballs, a block with incongruent-eligible oddballs (where the semantic meaning of the word represented a possible response; e.g., the word RED printed in green, with "red" as a possible response), and a block with incongruentineligible oddballs (e.g., the word PURPLE printed in green, when purple was not a possible response). This fMRI study established activity within the left DLPFC (BA 6/9) during all three types of oddball trial relative to the baseline neutral trials. This indicates that the presentation of infrequent trials increases the need for cognitive control. Thus, it seems that a temporary increase in the attentional set is not restricted to when conflict is expected, but might instead reflect when working memory is relied on for fast, task-relevant representations. On the other hand, the right DLPFC (BA 46) activated in Milham et al.'s study during all incongruent trials, both frequently and infrequently occurring. This suggests that the right DLPFC is not related to an attentional set increase due to online use of working memory following an unexpected but conflicting trial.

Likewise, in a recent fMRI study, Liu, Bai, and Zhang (2008) used a Stroop color-word task consisting of highconflict blocks (approximately 70\% congruent, 30\% incongruent trials) and low-conflict blocks (approximately $30 \%$ congruent, $70 \%$ incongruent trials). Their findings showed a correlation between right DLPFC activity (BA 46) and a high ratio of either congruent or incongruent trials in both blocks, which can be associated with macroadjustments due to an overall increased attentional set for frequent stimuli, but no such correlation for left DLPFC. This implies that the right DLPFC is not strictly related to the expectation of conflict, but to context-driven regulation and the executive modification of cognitive control.

Within this context, Vanderhasselt et al. (2007) investigated the influence of rTMS on a Stroop task in which participants had to switch between word reading and color naming within a low-conflict ( $80 \%$ incongruent trials) and a medium-conflict (50\% incongruent) condition. This 
Stroop paradigm was a combination of a task-switching Stroop version with variation of the ratio of congruent and incongruent trials. After one session of high-frequency $(10 \mathrm{~Hz})$ rTMS over the right DLPFC (BA 9/46), decreased color-naming RTs occurred solely in the low-conflict condition, whereas performance after the word-reading instruction was not influenced (no general speeding effects). In the medium-conflict condition, in which conflict on incongruent trials was not decreased by cognitive control, latencies were not affected for either color naming or word reading. This is in line with our assumption that the right DLPFC is related to macro-adjustments in situations of overall conflict.

Unpublished data from left DLPFC (BA 9/46) stimulation using the same design revealed decreased RTs for color naming in both medium- and low-conflict conditions, whereas no effects were found after the word-reading instruction (Vanderhasselt, De Raedt, Leyman, \& Baeken, 2009). These results are in line with the left, but not the right, DLPFC being related to a preparatory and temporary increased attentional set.

\section{Conflict Adaptation}

The third Stroop variant for manipulating cognitive control is measuring conflict adaptation effects (sequential effects) in blocks with equal numbers of congruent and incongruent trials.

Egner and Hirsch (2005) identified, using fMRI, neural substrates in the right DLPFC (BA 46) of conflict adaptation effects during a variant of the Stroop task in which face stimuli were used as either target or distractor stimulus features. Participants had to categorize famous faces on the basis of whether they belonged to an actor or a politician, while trying to ignore category-congruent or -incongruent names written across those faces (e.g., an actor's face with a politician's name). To distinguish between conflict- and control-related processes in this Stroop-like paradigm, Egner and Hirsch combined equal proportions of the following sequences: a congruent trial after a congruent trial, an incongruent after a congruent trial, a congruent trial after an incongruent trial, and an incongruent trial after another incongruent trial. For incongruent trials, they identified more activation in the right DLPFC (BA 46) after other incongruent trials than after congruent trials, which represented context-specific conflict resolution through cognitive control. The possible explanation of categorypriming effects was excluded, because the stimulus congruency effects were controlled for stimulus probability and repetition priming effects.

These results were replicated in a very similar study by Egner, Etkin, Gale, and Hirsch (2008), in which the resolution of nonemotional conflict was exclusively associated with activity in right DLPFC (BA 9).

Moreover, Kerns et al. (2004) demonstrated that trials exhibiting fast RTs following conflict (after incongruent trials and after stimulus repetitions were removed), as well as posterror trials, were associated with increased activity in the right DLPFC (BA 9/46). These fMRI findings support the theory that the right DLPFC is particularly relevant for top-down modulation and for control implementation when building representations that bias behavior toward an appropriate response or set of features (Blasi et al., 2006). In addition, Kerns et al. established a positive correlation between Stroop accuracy and activation in the right DLPFC, and they suggested that response conflict engaged right DLPFC. Within this context, the right DLPFC seemed to be specifically implemented in conditions in which attentional control was elicited for reducing conflict (Kerns et al., 2004).

\section{Conclusions}

These research findings provide support for the theory that the DLPFC (BA 9/46) mediates cognitive control to prevent future conflicts through an increased attentional set for task-relevant information.

Notwithstanding this commonly accepted account, here we have highlighted some specific aspects of DLPFC lateralization. Kerns et al. (2004) explained the heterogeneous research findings concerning lateralization in terms of the different task configurations used in the various studies. At present, the data available in the literature allow for a lateralization of processes in the DLPFC during Stroop task performance.

It has been suggested that the left DLPFC is activated by distractor incongruency, because of its increased activation during incongruent Stroop trials (e.g., Banich et al., 2000; Milham et al., 2003). Extending these theoretical accounts, the left DLPFC seems to be activated during both congruent and incongruent trials, particularly during times of high conflict with the context representations (i.e., the color instruction; see, e.g., MacDonald et al., 2000). Specifically, when cognitive control should temporally be increased because of varying instructions, the left DLPFC seems to have an influence on preparatory attentional set representations. The left DLPFC seems related to active preparatory task correlates of cognitive control that require rapid maintenance of online representations. This suggests the involvement of the left DLPFC with working memory through the coordinated recruitment of brain systems, via attention, to achieve higher cognitive skills (see Postle, 2006).

On the other hand, the right DLPFC seems related to online processes of cognitive control to reduce subsequent attentional conflict. This implies a postconflict recruitment of the right DLPFC to subsequently minimize conflict. In Stroop tasks, this entails that RTs on incongruent trials are faster specifically after other incongruent trials and are associated with increased right DLPFC activity. Moreover, the right DLPFC is selectively activated during frequent congruent and incongruent Stroop trials, because of macro-adjustments of cognitive control.

Overall, these findings suggest that different upregulation mechanisms are activated in the left and right hemispheres. Left DLPFC activation seems related to contexts in which a temporal up-regulation of the attentional set is required. These attentional adjustments are not based on the amount of conflict, but on participants' expectancies regarding the nature of the upcoming trial 
(Egner, 2007). The right DLPFC, on the other hand, seems related to online macro-adjustments in a conflict-driven context, resulting in an up-regulation of cognitive control. This reduces the interference of an irrelevant stimulus dimension with performance, resulting in a decreased congruency effect following incongruent trials or in a context of frequent conflicting trials.

\section{AUTHOR NOTE}

M.-A.V. is a postdoctoral fellow of the Research Foundation, Flanders (FWO). This study was also supported by a grant from the Scientific Fund W. Gepts, University Hospital, Free University of Brussels (AZ-VUB). Correspondence related to this article may be sent to M.-A. Vanderhasselt, Department of Psychology, Ghent University, Henri Dunantlaan 2, B-9000 Ghent, Belgium (e-mail: marieanne.vanderhasselt@ugent.be).

\section{REFERENCES}

Aarts, E., Roelofs, A., \& van Turennout, M. (2008). Anticipatory activity in anterior cingulate cortex can be independent of conflict and error likelihood. Journal of Neuroscience, 28, 4671-4678.

Banich, M. T., Milham, M. P., Atchley, R. A., Cohen, N. J., Webb, A., Wszalek, T., ET AL. (2000). Prefrontal regions play a predominant role in imposing an attentional "set": Evidence from fMRI. Cognitive Brain Research, 10, 1-9.

BARber, A. D., \& CARTER, C. S. (2005). Cognitive control involved in overcoming prepotent response tendencies and switching between tasks. Cerebral Cortex, 15, 899-912.

Blasi, G., Goldberg, T. E., Weickert, T., Das, S., Kohn, P., Zoltick, B., ET AL. (2006). Brain regions underlying response inhibition and interference monitoring and suppression. European Journal of Neuroscience, 23, 1658-1664.

Botvinick, M. M., Braver, T. S., Barch, D. M., Carter, C. S., \& Cohen, J. D. (2001). Conflict monitoring and cognitive control. Psychological Review, 108, 624-652.

Botvinick, M. [M.], Nystrom, L. E., Fissell, K., Carter, C. S., \& Cohen, J. D. (1999). Conflict monitoring versus selection-for-action in anterior cingulate cortex. Nature, 402, 179-181.

EgNER, T. (2007). Congruency sequence effects and cognitive control. Cognitive, Affective, \& Behavioral Neuroscience, 7, 380-390.

Egner, T., EtKin, A., Gale, S., \& Hirsch, J. (2008). Dissociable neural systems resolve conflict from emotional versus nonemotional distracters. Cerebral Cortex, 18, 1475-1484.

EgNer, T., \& Hirsch, J. (2005). Cognitive control mechanisms resolve conflict through cortical amplification of task-relevant information. Nature Neuroscience, 8, 1784-1790.

Gratton, G., Coles, M. G. H., \& Donchin, E. (1992). Optimizing the use of information: Strategic control of activation of responses. Journal of Experimental Psychology: General, 121, 480-506.
Harrison, B. J., Shaw, M., YÜcel, M., Purcell, R., Brewer, W. J., Strother, S. C., ET AL. (2005). Functional connectivity during Stroop task performance. Neurolmage, 24, 181-191.

Kerns, J. G., Cohen, J. D., MacDonald, A. W., III, Cho, R. Y., Stenger, V. A., \& CARTER, C. S. (2004). Anterior cingulate conflict monitoring and adjustments in control. Science, 303, 1023-1026.

LiU, J., BaI, J., \& Zhang, D. (2008). Cognitive control explored by linear modelling behaviour and fMRI data during Stroop tasks. Physiological Measurement, 29, 703-710.

MacDonald, A. W., III, Cohen, J. D., Stenger, V. A., \& Carter, C. S. (2000). Dissociating the role of the dorsolateral prefrontal and anterior cingulate cortex in cognitive control. Science, 288, 1835-1838.

Milham, M. P., Banich, M. T., \& Barad, V. (2003). Competition for priority in processing increases prefrontal cortex's involvement in topdown control: An event-related fMRI study of the Stroop task. Cognitive Brain Research, 17, 212-222.

Miller, E. K., \& Cohen, J. D. (2001). An integrative theory of prefrontal cortex function. Annual Review of Neuroscience, 24, 167-202.

PostLe, B. R. (2006). Working memory as an emergent property of the mind and brain. Neuroscience, 139, 23-38.

Rubia, K., Smith, A. B., Woolley, J., Nosarti, C., Heyman, I., TayLOR, E., \& Brammer, M. (2006). Progressive increase of frontostriatal brain activation from childhood to adulthood during event-related tasks of cognitive control. Human Brain Mapping, 27, 973-993.

STROOP, J. R. (1935). Studies of interference in serial verbal reactions. Journal of Experimental Psychology, 18, 643-662.

Stürmer, B., Redlich, M., Irlbacher, K., \& Brandt, S. (2007). Executive control over response priming and conflict: A transcranial magnetic stimulation study. Experimental Brain Research, 183, 329-339.

Stuss, D. T., Floden, D., Alexander, M. P., Levine, B., \& Katz, D. (2001). Stroop performance in focal lesion patients: Dissociation of processes and frontal lobe lesion location. Neuropsychologia, 39, 771786.

Swick, D., \& Jovanovic, J. (2002). Anterior cingulate cortex and the Stroop task: Neuropsychological evidence for topographic specificity. Neuropsychologia, 40, 1240-1253.

Vanderhasselt, M.-A., De Raedt, R., Baeken, C., Leyman, L., ClerinX, P., \& D'Haenen, H. (2007). The influence of rTMS over the right dorsolateral prefrontal cortex on top-down attentional processes. Brain Research, 1137, 111-116.

Vanderhasselt, M.-A., De Raedt, R., Baeken, C., Leyman, L., \& D'HAENEN, H. (2006). The influence of rTMS over the left dorsolateral prefrontal cortex on Stroop task performance. Experimental Brain Research, 169, 279-282.

Vanderhasselt, M.-A. De Raedt, R., Leyman, L., \& Baeken, C. (2009). The influence of rTMS over the left dorsolateral prefrontal cortex on Stroop task performance. Unpublished data.

(Manuscript received November 21, 2008; revision accepted for publication January 27,2009 .) 